\title{
Fissuras no pavimento da rodovia ERS-149: Geofísica aplicada como auxílio na caracterização do terreno
}

Marieli Machado Zago', Maximilian Fries¹, Igor Magalhães Clemente' ${ }^{1}$, Elisa Zago Porto², Andrea Valli Nummer², Rinaldo José Barbosa Pinheiro²

'Laboratório de Geofísica Aplicada (LGA) Unipampa, Campus Caçapava do Sul; ²Universidade Federal de Santa Maria

Copyright 2019, SBGf - Sociedade Brasileira de Geofísica

This paper was prepared for presentation during the $16^{\text {th }}$ International Congress of the Brazilian Geophysical Society held in Rio de Janeiro, Brazil, 19-22 August 2019.

Contents of this paper were reviewed by the Technical Committee of the $16^{\text {th }}$ International Congress of the Brazilian Geophysical Society and do not necessarily represent any position of the SBGf, its officers or members. Electronic reproduction or storage of any part of this paper for commercial purposes without the written consent of the Brazilian Geophysical Society is prohibited.

\section{Abstract}

Geophysics is widely recognized tool in indirect subsurface and non-invasive investigation. It is applied in different near-surface and geotechnical studies. As an example, we can mention ground investigations for structures, cables, fractures, cavities and water infiltration. In geotechnics, lithologies heterogeneities, fault/joints and basement rocks soil (altered zone) investigations have been producing significant results with low costs, less time and simple logistics. This study proposes the use of direct current resistivity profiles to detect and characterize altered rocks layers limits (regolith), water saturated areas and depth as an auxiliary aid to characterize a collapsed asphalt paved road (ERS-149). The area is located between Faxinal do Soturno and Nova Palma cities, Rio Grande do Sul State, Brazil. Locally, on the road side, several cracks occurred due the ground instability leading to an asphalt and material below collapse. We performed two DC-resistivity profiles perpendicular to the road integrated with a high-resolution digital elevation model. The results presented a large amount of unconsolidated material (soil and rocks fragments) and a significant inpore water filling zones. These features, associated with the gravitational action in the area are interpreted as the main sliding, cracking and collapsing causes. The use of geophysics in this context was a valuable and effective method as an aid to conduct and locate direct subsurface investigations as detail geological and soil mapping and drill-holes to better describes the lithology/materials refining the subsurface modeling.

\section{Introdução}

Os métodos geofísicos estão sendo cada vez mais utilizados na engenharia civil, principalmente em fases preliminares de projetos que demandam investigações de subsuperfície para a construção de pavimentações ou edificações. Algumas problemáticas envolvendo mecânica de rochas, geologia de engenharia, engenharia geotécnica, água subterrânea, meio ambiente ou de pavimentos são solucionadas com mais eficácia e baixo custo a partir dessa ferramenta. Como exemplo, possibilita reduzir o número de sondagens convencionais devido ao auxílio na localização de pontos críticos de interesse. Consequentemente, diminui-se significantemente os custos de um projeto (Abidin et al., 2011). Crawford et. al., (2017), utilizou o método de resistividade elétrica em deslizamentos coluviais superficiais para auxiliar na interpretação de falhas de escorregamentos e características dos limites litológicos, espessura do solo e na identificação de zonas saturadas e insaturadas e, também, nas variações de umidade. Henaish e Attwa (2018) fizeram um mapeamento estrutural detalhado, com o auxílio do método geofísico de resistividade elétrica para estudar as condições de geo-engenharia de uma estrada de terra.

O método geofísico de eletrorresistividade consiste na análise e interpretação do parâmetro físico de resistividade elétrica obtido a partir de medidas efetuadas na superfície do terreno investigando ao longo de uma seção a sua variação na horizontal, a uma ou mais profundidades determinadas (Orellana, 1972). Empregase o uso de uma fonte de corrente e um amperímetro que mede a intensidade da corrente introduzida no solo através dos eletrodos de corrente para que se estabeleça um campo elétrico em subsuperfície. O campo elétrico gerado é analisado por meio de um par de eletrodos de potencial conectados à um instrumento capaz de medir a diferença de potencial (Telford et al., 1990).

Uma técnica muito empregada denomina-se Caminhamento Elétrico (CE). Esta, tem como objetivo detectar e localizar contatos verticais, falhas, diques, zonas de cisalhamento e veios com mergulho acentuado considerando o contraste de condutividade/resistividade apresentado (Orellana, 1972). Para a aquisição de dados em campo é preciso definir o arranjo a ser executado em campo, ou seja, a disposição dos eletrodos no desenvolvimento da técnica. Existem várias modalidades de arranjos utilizados em campo, denominados de Wenner, Wenner Shulumberger, Dipolo Dipolo, Polo Dipolo, dentre outras. Diferem entre si principalmente na profundidade de investigação, cobertura de análise em subsuperfície, razão sinal/ruído e tempo de leitura em campo.

O presente estudo teve como objetivo utilizar o método geofísico de eletrorresistividade no auxílio a caracterização de contatos entre diferentes materiais rochosos como solo e zonas saturada/insaturada de água. A área de estudo localiza-se próximo a uma rodovia onde ocorreu a abertura de fendas/fissuras na pavimentação asfáltica em decorrência da instabilidade do terreno. Foram realizados perfis de eletrorresistividade integrados a um modelo digital de elevação possibilitando identificar-se zonas de material inconsolidado (rocha alterada e fragmentos) e sujeitas a mobilidade 
desencadeando um alívio de pressão nas margens da rodovia e consequente colapsamento no local.

\section{Área de estudo}

Localiza-se as margens da rodovia ERS 149 entre os municípios de Faxinal do Soturno e Nova Palma, Estado do Rio Grande do Sul, Brasil. A oeste da rodovia encontra-se o rio Soturno localizado paralelamente a rodovia (a oeste da ERS 149, Figura 1a). A leste da rodovia há significante contraste topográfico com altitude variando de 70 a 156 metros (Figura 1b). A sudoeste, há um açude com significante volume de água.

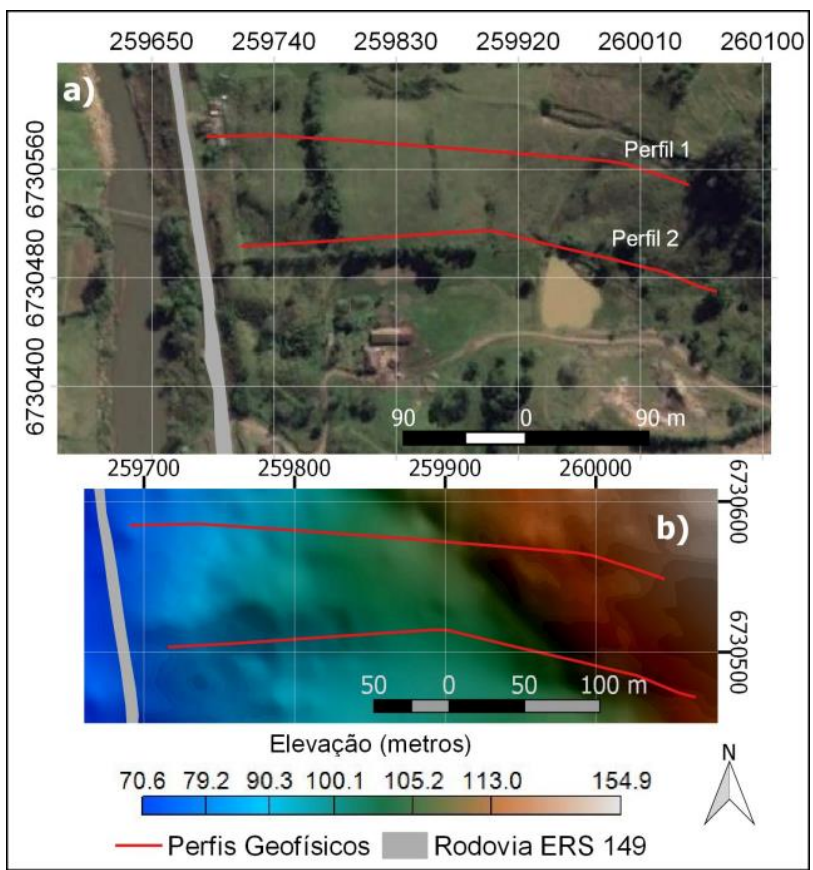

Figura 1 - Mapa de localização da área de estudo com os perfis em vermelho. Em a) sobreposto a imagem de satélite e em b) sobreposto ao modelo digital de elevação (MDE). Fonte: modificado de Alos Palsar, 2018.

Na figura 2 observa-se a encosta onde foram adquiridos dados geofísicos logo abaixo um trecho da rodovia ERS 149 (linha amarela), demarcado com uma elipse vermelha a zona de ruptura da rodovia e um retângulo azul representando a localização de um trecho do rio Soturno.

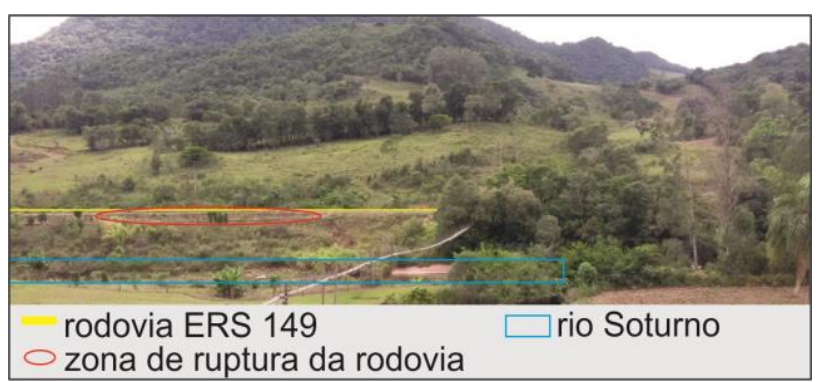

Figura 2 - Encosta onde foi realizada a campanha geofísica e demarcações de localização da rodovia ERS 149 , zona de ruptura da rodovia e o rio Soturno.
Localmente a área de estudo é formada por arenitos finos com lentes silto-argilosas da Formação Caturrita (onde está assentado o aterro da rodovia) e sobre elas ocorre os arenitos da Formação Botucatu. Próximo ao perfil 2 encontram-se basaltos da Formação Serra Geral, intercalados a estes, ocorre os arenitos da Formação Botucatu.

A encosta é recoberta por colúvios que são compostos de materiais de matriz areno-argilosa com blocos de rochas de diversos tamanhos. Na base da encosta, às margens do rio Soturno, encontram-se depósitos alúvio/coluvionares.

\section{Materiais e métodos}

Para o desenvolvimento do estudo planejou-se a aquisição de dados geofísicos com base em informações preliminares de relevo, litologias e localização das fendas/fissuras na pavimentação asfáltica. Para a aquisição de dados no campo foram utilizados 4 multicabos de 90 metros de extensão, 72 eletrodos e um resistivímetro modelo Syscal Pro fabricado pela Iris Instruments.

Foi adotado uma sequência geométrica de arranjo de eletrodos, com o uso do software Prosys II (Iris Instruments). A fim de se atingir uma profundidade teórica de investigação de aproximadamente 80 metros (profundidade) foram adquiridos dois perfis. O arranjo selecionado para a execução dos perfis foi o Polo Dipolo, programado para 7 níveis de investigação. Para o desenvolvimento das linhas em campo, 4 multicabos de 90 metros cada foram utilizados. Um total de 360 metros de extensão foi obtido com os eletrodos dispostos a cada 5 metros ao longo de toda a extensão arranjo. $O$ equipamento Syscal (unidade de aquisição) ficou disposto no meio do arranjo. Para o arranjo polo dipolo é preciso que um eletrodo seja colocado a uma distância suficientemente longe da linha do levantamento (Loke, 2000). Essa distância deve corresponder a pelo menos dez vezes a maior abertura entre os eletrodos extremos do arranjo que permanecem na linha do levantamento (Keller \& Frischknecht, 1966; Telford et. al, 1990), assim assegura-se que ocorreu pouca influência sobre o resto do arranjo. Neste estudo utilizou-se um fio elétrico com 500 metros de extensão e constatou-se uma qualidade de dados considerável. (Figura 3).

$\begin{array}{ll}\text { - Multicabos de } 90 \text { metros } & \text { Resistivimetro Syscal Pro } \\ \text { - Caixas de conexão } & \text { Fio elétrico de } 500 \text { metros } \\ \text { - Bateria } 12 \text { Volts } & \end{array}$

Figura 3 - Esquema demonstrativo do desenvolvimento dos perfis geofísicos no campo.

\section{Resultados e discussões}

Após a aquisição dos dados em campo, os perfis foram pré-processados e fez-se a inserção da topografia em cada perfil. Para as medidas dos valores de resistividade elétrica das pseudoseções foi realizado o processo de inversão dos valores de resistividade elétrica por meio do software Res2Dinv versão 3.4 da Geotomo Inc. O arranjo polo dipolo apresentou-se com baixa razão sinal/ruído e com uma profundidade de investigação de aproximadamente 80 metros. A escala de cores 
selecionada para os valores de resistividade varia de 1.6 ohm.m a 657 ohm.m conforme a Figura 4.

Nos perfis 1 e 2 foram considerados baixos anômalos os valores de 1,6 a 20,9 ohm.m; anomalias intermediárias com uma variação de 21 a 150 ohm.m e altos anômalos de 150 a 277 ohm.m.

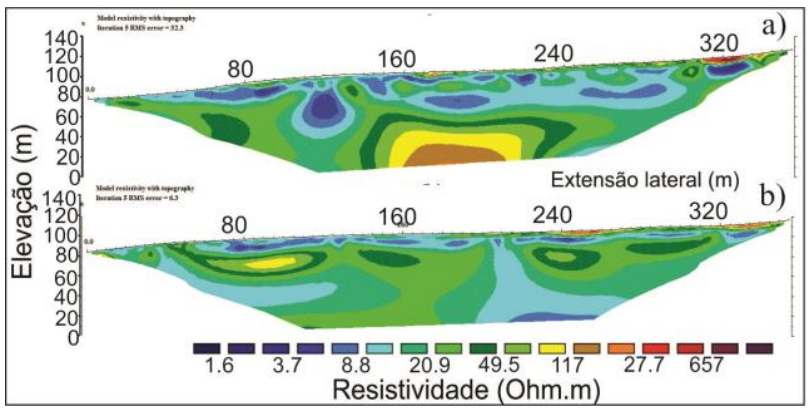

Figura 4 - Seções de resistividade elétrica realizadas no estudo em a) Perfil 1 e em b) Perfil 2.

Para análise e interpretação das seções de resistividade elétrica utilizou-se os propostos por Telford et al. (1990). Estes, apresentam valores de referência de resistividade elétrica para rochas e materiais presentes na área estudada (Tabela 1).

\begin{tabular}{cc}
\hline Material & $\begin{array}{c}\text { Resistividade Elétrica } \\
\text { (Ohm.m) }\end{array}$ \\
\hline $\begin{array}{c}\text { Água em subsuperfície } \\
\text { (Rochas sedimentares) }\end{array}$ & $10-100$ \\
Arenitos & $1-6.4 \times 10^{8}$ \\
Aluvião e areias & $10-800$ \\
\hline
\end{tabular}

Tabela 1 - Valores de resistividade elétrica dos materiais. Fonte: Extraído de Telford et. al., 1990.

Os baixos anômalos de resistividade elétrica são correlacionáveis a uma zona fortemente saturada. Os materiais de resistividade intermediária são interpretados como aluvião e arenito insaturado e os altos anômalos interpretados como uma zona fortemente insaturada (Figura 5).

No perfil 1 observou-se uma zona fortemente saturada em toda a extensão do perfil de aproximadamente 20 metros de profundidade. Esta área correlaciona-se a materiais (solos/fragmentos de rocha) com menos resistência e mais sujeito a mobilidades por meio de ações gravitacionais e intempéricas. Na porção central do perfil observa-se um corpo rochoso de aproximadamente 40 metros de largura e 100 metros de comprimento, localizado de 20 a 60 metros de profundidade. Estes altos anômalos representam materiais mais consolidados com menos possibilidades de alterações ou deslocamentos (menor permeabilidade) (Figura 5a).

No perfil 2 baixos anômalos superficiais foram detectados, contendo aproximadamente 10 metros de espessura e 160 metros de comprimento. Em aproximadamente 210 metros de extensão do perfil, estes baixos resistivos atingem a profundidade máxima de investigação e paralelo a linha onde se localiza o açude (Figura 1). Observou-se, também, a presença de materiais mais consolidados, solos ou fragmentos de rochas, (anomalias intermediárias a altas) dispostos ao longo de todo o perfil. Estes, são menos suscetíveis a sofrer alterações e deslocamentos. Os baixos anômalos mais profundos são característicos de possíveis fraturamentos locais, por onde ocorre a percolação de fluídos e consequente recarga do açude (Figura 5b).

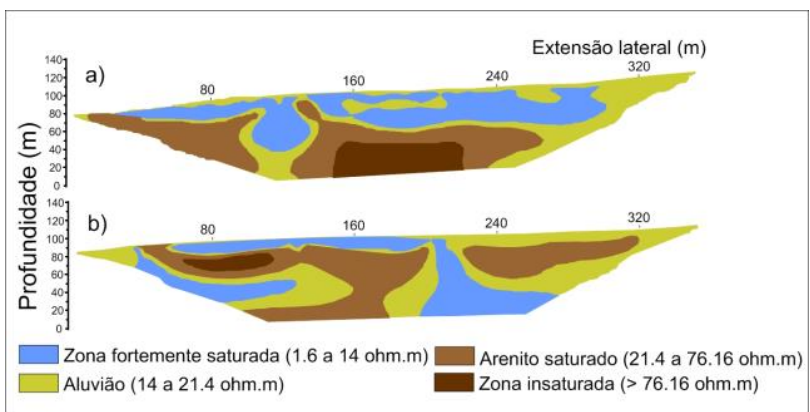

Figura 5 - Seções elétricas interpretadas em a) perfil 1 e em b) Perfil 2.

Foram geradas visualizações tridimensionais (3D) das seções de resistividade elétrica integradas a um modelo digital de elevação (MDE) conforme apresentado na Figura 6 . A Figuras 6 a e b oferece uma visão geral da área de estudo com o Rio Soturno na porção oeste (W), em paralelo com a rodovia ERS 149 e as duas seções dispostas paralelamente a leste $(E)$ da rodovia.

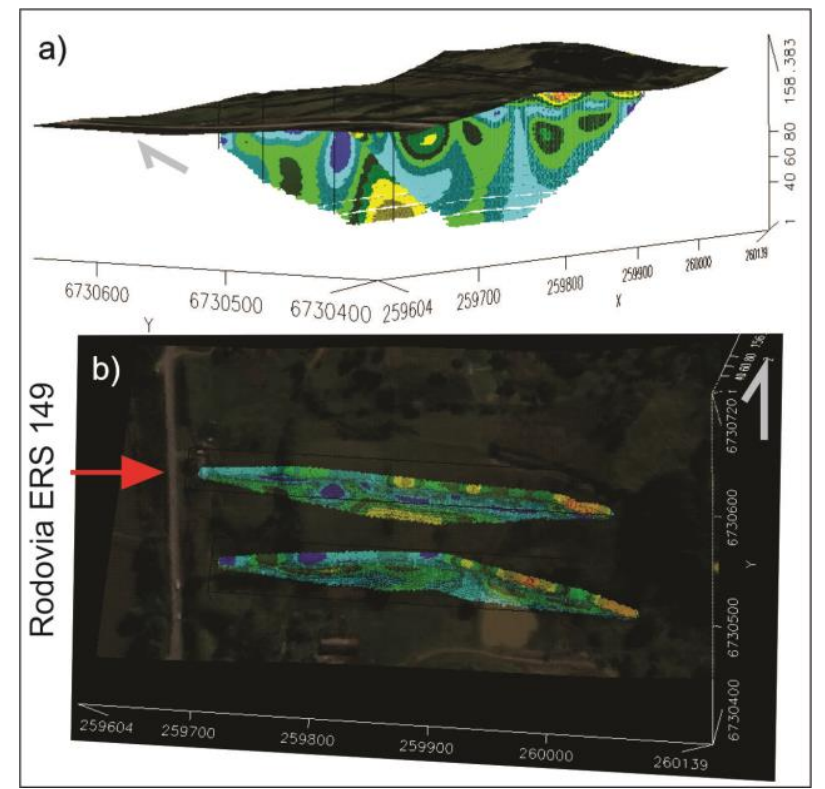

Figura 6 - Visualizações 3D das seções de eletrorresistividade. Em a) visão em diagonal dos perfis, em b) visão em planta da superfície topográfica com transparência de $60 \%$.

O terreno tem uma variação altimétrica de aproximadamente 40 metros no sentido W-E sendo a superfície de descolamento acentuada em decorrência da topografia, facilitando assim a movimentação de materiais menos consolidados.

$\mathrm{Na}$ Figura 7 os perfis 1 e 2 foram gerados com valores de resistividade elétrica limitados de 1.6 a 20 Ohm.m. A Figura $6 \mathrm{a}$, correspondente ao perfil 1 onde observa-se 
altos volumes de baixos resistivos. O perfil encontra-se próximo ao local de ruptura da rodovia. O perfil 2 (Figura 7b) apresentou uma densidade de valores de resistividade menor próximo a superfície, em contrapartida, tem-se baixos resistivos em profundidades mais acentuadas.

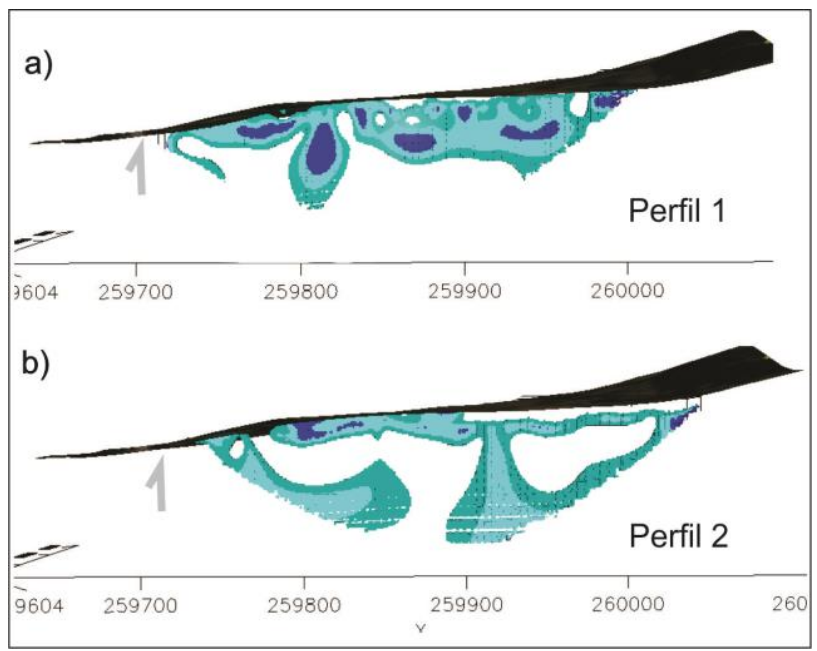

Figura 7 - Perfil 1 (a) e perfil 2 (b) com limites de resistividade elétrica de 1.6 a 20 ohm.m.

$\mathrm{Na}$ Figura 8 os perfis 1 e 2 possuem valores de resistividade elétrica de 20 a 300 ohm.m. De acordo com a interpretação das seções da Figura 4, estes são compostos por aluvião, arenito saturado e zona insaturada. Estes materiais podem proporcionar uma resistência e consequentemente auxiliar na fixação de materiais em superfície. Observa-se que no perfil 1 (Figura 8a) ocorre uma densidade de altos resistivos profundos apenas na porção central do perfil. No perfil 2 (Figura 8b), maiores áreas com altos resistivos ao longo de todo o perfil são observados.

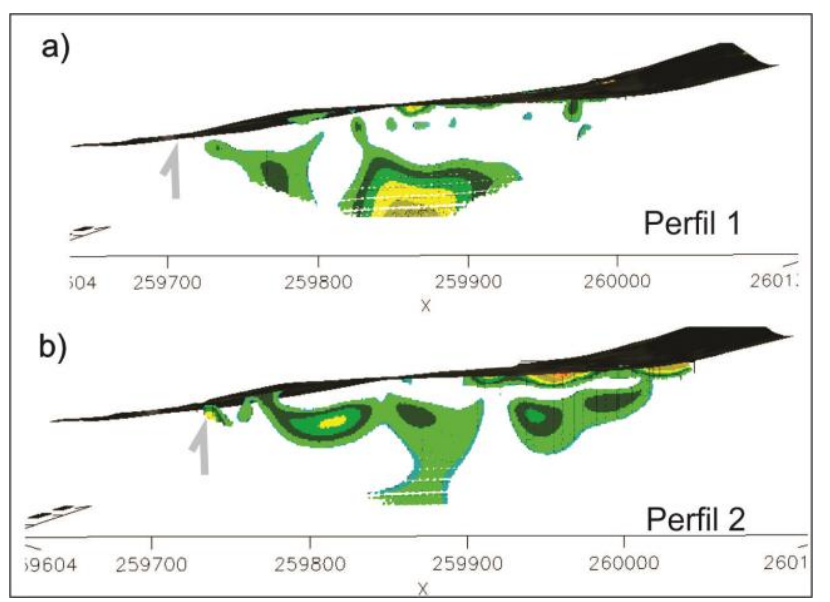

Figura 8 - Perfil 1 (a) e perfil 2 (b) com limite de resistividade elétrica de 20 a 300 ohm.m.

No esquema da Figura 9 apresenta-se os perfis 1 e 2 localizados em uma imagem da superfície com uma transparência suave juntamente com a rodovia ERS 149 (linha vermelha), o rio Soturno (linha azul) e um círculo indicando a localização da área crítica com alteração do pavimento asfáltico da rodovia (abertura de fendas e fissuras).

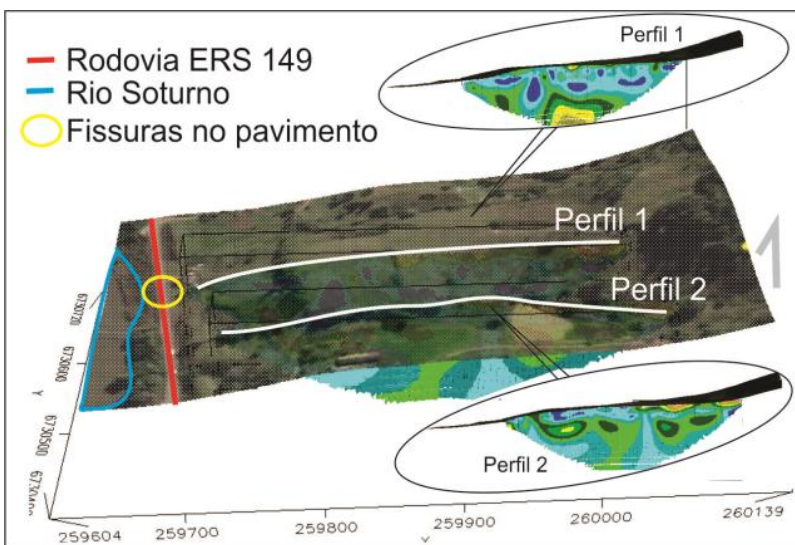

Figura 9 - Esquema com os perfis 1 e 2 de eletrorresistividade, a rodovia ERS 149, o rio soturno e a indicação de zonas com fissuras no pavimento.

A partir das demarcações dos limites de resistividade elétrica (Figuras 7 e 8) foi possível interpretar que o perfil 1 demonstrou uma instabilidade maior, devido a grande densidade de baixos resistivos correlacionados com zonas insaturadas. Observa-se (Figura 9) que o perfil 1 está localizado as margens da rodovia ERS 149 e exatamente onde há ocorrências de rupturas no asfalto. A combinação de um terreno com uma elevação topográfica gradual para leste e significante densidade de baixos resistivos pode ser diretamente associado as alterações na rodovia e consequente colapsamento. Zonas insaturadas facilitam a mobilidade de massas em diferentes níveis de profundidade e somadas a alta declividade no terreno pode ser um importante elemento na abertura de fendas e fissuras no local.

A aquisição do perfil 2 foi feita em uma área onde não há fissuras no pavimento asfáltico a fim de comparar-se os padrões de resistividade no estudo. Ao longo deste, é visível uma maior densidade de altos resistivos correlacionados com zonas saturadas sendo estas características de uma maior fixação do solo no local, dificultando a mobilidade de massas.

\section{Conclusão}

O uso da geofísica aplicada por meio do método da eletrorresistividade mostrou-se satisfatório como ferramenta auxiliar na análise e interpretação prévia de causas do colapsamento em uma rodovia asfaltada. A partir da interpretação das seções de resistividade elétrica identificou-se zonas saturadas e insaturadas e diferentes contatos litológicos. A integração dos resultados a um Modelo Digital de Elevação (MDE) da área de estudo possibilitou identificar variações topográficas acentuadas nas margens da rodovia. Estas, são associadas ao caráter estrutural do local (falhas e fraturas), influenciando os valores de resistividade e permitindo um refinamento do modelo geológico estrutural em estudos futuros.

Em decorrência da quantidade de baixos resistivos do perfil 1 conclui-se que, próximo ao local, há menos 
resistência de solo estando mais sujeitos a alterações. Desta forma, o deslocamento de materiais inconsolidados (solos/fragmentos de rocha) a partir de ações intempéricas e gravitacionais são intensificados. Estes materiais podem desencadear um processo de pressão do topo para a base das camadas (no sentido E-W), ocasionando o colapsamento da rodovia devido ao alívio de pressão em baixos topográficos (onde está localizada a rodovia). No perfil 2 não foram identificados grandes volumes de baixos resistivos concentrados.

\section{Referências}

ALOS PALSAR - Modelo Digital de Elevação com resolução de 12,5 metros. Vertex, portal de dados do Alaska Satellite Facility's para download de imagens de sensoriamento remoto, 2018. Disponível em: $<$ https://vertex.daac.asf.alaska.edu/>. Acesso em: 28 agosto de 2018.

ABIDIN, M. H. Z; SAAD. R; AHMAD, F; WIJEYESEKERA, D. C; BAHARUDDIN, M. F. T. Application of Geophysical Methods in Civil. Anais... In: Engineering Malaysian technical universities international conference on engineering \& technology. MUICET, 2011. MUICET. 2011.

CRAWFORD, M. M., BRYSON, L. S., WOOLERY, E. W., WANG, Z. Using 2-D electrical resistivity imaging for joint geophysical and geotechnical characterization of shallow landslides.

Appgeo, doi:10.1016/j.jappgeo.2018.06.009, 2017.

2017

HENAISH, A., ATTWA, M. Internal structural architecture of a soft-linkage transfer zone using outcrop and DC resistivity data: Implications for preliminary engineering assessment. Engeo, 2018, doi:10.1016/j.enggeo.2018.07.018, 2018

KELLER, G. V., \& FRISCHKNECHT, F. C. 1966. Electrical methods in geophysical prospecting. Oxford: Pergamon Press. 517p.

LOKE, M. H. 2000. Eletrical imaging surveys for environmental and engineering studies: A pratical Guide to 2-D and 3-D surveys.

ORELLANA, E. Prospección Geoeletrica en corriente continua. Editora Paraninfo $2^{2}$ edição. Madrid. 1972.

TELFORD, W. M., GELDART, L. P., SHERIFF, R. E. Applied Geophysics. Editora Cambridge $2^{\underline{a}}$ edição. NY. 1990. 